\title{
Precipitate-dislocation interaction mediated Portevin-Le Chatelier-like effect in a beta-stabilized Ti-Mo-Nb-Al alloy
}

\author{
D. Choudhuri ${ }^{1}$, S.A. Mantri ${ }^{1}$, T. Alam ${ }^{1}$, S. Banerjee ${ }^{3}$ and R. Banerjee ${ }^{1,2 *}$ \\ ${ }^{1}$ Department of Materials Science and Engineering, University of North Texas, Denton, \\ TX, 76191, USA \\ ${ }^{2}$ Center for Advanced Research and Technology, University of North Texas, Denton, \\ TX, 76191, USA \\ ${ }^{3}$ Bhabha Atomic Research Centre, Mumbai, India \\ * Corresponding author: Raj.Banerjee@unt.edu
}

\begin{abstract}
Portevin-Le Chatelier (PLC)-like effect was observed in a commercial alloy based on TiMo-Nb-Al, which exhibited serrations in its tensile stress-strain response within a temperature-strain rate window. The serrations manifested as repeated load-drop and rise, and were rationalized on the basis of sudden plastic flow along the "soft" $\omega$ precipitate free micro-slip-bands created by the passage of leading dislocations in the parent $\beta$ matrix. Subsequently, dynamic precipitation of the $\alpha$ phase occurred within the $\omega$-free micro-slip-bands. These results demonstrate that the PLC-like effect can be manifested via precipitate-dislocation interactions, and is not limited to the traditionally accepted solute-dislocation interactions.
\end{abstract}




\section{Main-text}

The Portevin-Le Chatelier (PLC) effect typically manifests itself as a serrated plasticflow comprising successive occurrences of load-drop and rise in the uniaxial stress-strain response of metallic alloys, within a strain rate - temperature window. [1-4]. Though the PLC phenomenon is ubiquitous (observed in $\mathrm{Fe}, \mathrm{Ti}, \mathrm{Al}$,-based alloys and intermetallics [1-6]), there is no unique mechanism for its occurrence. The common factors, responsible for discontinuous plastic flow in different systems are, sudden release of mobile dislocations followed by arresting them via a thermally activated pinning process $[1,2]$.

One of the earliest observations of PLC in Ti alloys was noted in commercially pure $h c p$ Ti [7], and recently in a commercial alpha ( $\alpha)$-Ti alloy designated as 834 [8]. Prasad et.al. demonstrated that PLC effect in $\alpha$-Ti alloys is manifested via pinning of dislocations by interstitial elements like $\mathrm{C}$ and $\mathrm{N}$ [8]-consistent with the established models [1]. In contrast to $\alpha$-Ti alloys, detailed studies of PLC effect in beta ( $\beta$ )-stabilized $\mathrm{Ti}$ and $\mathrm{Zr}$ alloys and Ti-Nb-Al intermetallics indicated that the PLC effect is influenced by the presence of the metastable omega $(\omega)$ phase in the matrix of $b c c-\beta$-phase $([3,6])$.

Discontinuous plastic flow in a $\beta$-Ti alloy was first observed in Ti-15wt\% Mo under different combinations of temperatures and strain rates $[3,4]$. These results indicated that the load-drop during PLC occurred due to the shearing of $\omega$ precipitates by dislocations, leading to the creation of $\omega$-free "soft channels" in the microstructure [3,4]. Similar $\omega$ free channels were also observed in a plastically deformed Ti-Nb-based alloy by Lai et.al [9] and in Ti-Nb-Al intermetallics ([3,6]). The sudden release of an avalanche of dislocations in soft channels was attributed to the observed load-drops. However, the mechanism of the subsequent load-rise due to "hardening" of the prior soft channels via re-pinning of mobile dislocations is yet to be established convincingly.[3,4,6]). "Dynamic reconstruction" of $\omega$ precipitates in the prior $\omega$-free soft channels was suggested as a plausible mechanism for the hardening process [4].

The alloy studied here is $\beta 21 \mathrm{~S}$, a $\beta$-stabilized titanium alloy, comparable to Ti- $15 \mathrm{wt} \% \mathrm{Mo}$ in terms of equivalent $\beta$ stabilizing alloying additions $[10,11]$. However, the presence of 
$\mathrm{Nb}, \mathrm{Al}$ and $\mathrm{Si}$, which influences the relative stabilities the competing $h c p-\alpha, b c c-\beta$ and hexagonal- $\omega$ phases, make the transformation and deformation behavior different from that of the binary Ti-15wt\%Mo alloy. One of the important commercial applications of $\beta 21 \mathrm{~S}$ is in the exhaust nozzles of jet engines [10,11], where operating temperatures may overlap that of the occurrence of PLC effect. In the past absence of suitable techniques did not allow TEM examination from the deformation bands. Consequently, formation of soft $\omega$-free channels and subsequent pinning by fresh precipitates could not be observed. Thus, plastic deformation behavior of the $\beta 21 \mathrm{~S}$ alloy at $300^{\circ} \mathrm{C}$ and on the detailed microstructure of the deformation bands was undertaken.

Alloy samples of composition (Ti-9Mo-5.4Al-1.6Nb-0.3Si in at\%), or $\beta 21 \mathrm{~S}$, were heat treated in three conditions: (a) $\beta$ solutionized ( $\beta$-soln) at $900^{\circ} \mathrm{C} / 30 \mathrm{mins}$ followed by quenching in water,(b) $\beta$-solutionized and aged at $350^{\circ} \mathrm{C} / 100 \mathrm{Hrs}$ to form well developed isothermal $\omega$ precipitates and (c) $\beta$-solutionized and aged in two stages, $350^{\circ} \mathrm{C} / 100 \mathrm{Hrs}$ and $600^{\circ} \mathrm{C} / 1 \mathrm{Hr}$ to form equilibrium $\alpha$ precipitates.. Mini-tensile test specimens (gauge length $\sim 3 \mathrm{~mm}$, width $\sim 1 \mathrm{~mm}$ and thickness $\sim 0.8-1 \mathrm{~mm}$ ) were machined from the heattreated samples and tensile tested with at a strain-rate of $10^{-5} \mathrm{~s}^{-1}$ inside an oven at $300^{\circ} \mathrm{C}$. Description of tensile-testing equipment is presented elsewhere [11,12]. Microstructural characterization was performed with FEI Tecnai $\mathrm{G}^{2}$ transmission electron microscope (TEM) operating at $200 \mathrm{kV}$ and FEI Nova-NanoSEM ${ }^{\mathrm{TM}}$ scanning electron microscope (SEM) and a FEI Nova NanoLab $200^{\mathrm{TM}}$ focused ion beam (FIB) was used for extracting site-specific analysis.

Engineering stress vs. strain plots in Fig.1(a) shows the plastic-flow behavior at $300^{\circ} \mathrm{C}$ of the three heat-treated conditions : (a) $\beta$-soln $\quad$ (b) $\beta$-soln $+350^{\circ} \mathrm{C} / 100 \mathrm{Hrs}$ and (c) $\beta$ soln $+350^{\circ} \mathrm{C} / 100 \mathrm{Hrs}+600^{\circ} \mathrm{C} / 1 \mathrm{Hr}$. The plots for (a) and (b) exhibited pronounced serrations consisting of repeated load-drop and rise (inset Fig.1(a)) - a strong indicator of the PLC-like effect. On the other hand such serrated behavior was not discernable in the plastic flow curve corresponding to Sample (c). These results demonstrate initial 
microstructures influence serrated plastic flow in $\beta 21 \mathrm{~S}$ at $300^{0} \mathrm{C}$ and at $10^{-5} \mathrm{~s}^{-1}$ strain rate.

The initial microstructures $\quad \beta$-soln, $\quad \beta$-soln $+350^{\circ} \mathrm{C} / 100 \mathrm{Hrs}, \quad \beta$ soln $+350^{\circ} \mathrm{C} / 100 \mathrm{Hrs}+600^{\circ} \mathrm{C} / 1 \mathrm{Hr}$ are depicted in Fig. 1(b), 1(c) and 1(d) respectively via dark-field TEM images (DFTEM), where the foil planes were oriented close to [011] $]_{\beta}$ zone axis. Additionally, the corresponding $[011]_{\beta}$ selected area diffraction pattern (SADP) for each microstructure is shown as insets in the lower right corner. The DFTEM of the $\beta$-soln (Fig1.b) microstructure consisted of predominantly $b c c \beta$-phase matrix with mottled contrast. The SADP exhibits diffuse intensity distribution (manifested as reciprocal lattice streaking) characteristic of quenched-in athermal omegalike displacement fluctuations [3]. Such a structure is expected in alloys close to $(\beta+\omega) /$ $\beta$ metastable phase boundary [3]. Specimens annealed at $350^{\circ} \mathrm{C} / 100$ hours showed welldeveloped coarse omega precipitates ( 20nm long) in Fig.1(c), and the corresponding SADP (inset) indicated the characteristic $\omega$ reflections with intensity maximas at $1 / 3$ and $2 / 3[\overline{2} 1 \overline{1}]_{\beta}$ locations [3]. In few cases, very fine-scale $\alpha$ platelets were also noted in the DFTEM (top-right inset); the presence of a few $\alpha$-precipitates was also detectable in the $[011]_{\beta}$ SADP as faint intensity maxima at $1 / 2[\overline{2} 1 \overline{1}]_{\beta}$ which also suggested that the $\alpha$ phase fraction is substantially lower than that of $\omega[3,14]$. Subsequent annealing at $600^{\circ} \mathrm{C}$ resulted in complete dissolution of the $\omega$ phase and precipitation of $\alpha$ plates (DFTEM in Fig.1d and SEM in top inset). The presence of only $\alpha$ preciptates was further confirmed in the corresponding $[011]_{\beta}$ SADP, which also showed distinct $1 / 2[\overline{2} 1 \overline{1}]_{\beta}$, the expected $\alpha / \beta$ Burgers orientation relation (OR) : $(011)_{\beta} \|(0001)_{\alpha}$ and $[\overline{2} 1 \overline{1}]_{\beta} \|[01 \overline{1} 0]_{\alpha}$, and the absence of $\omega$ reflections at $1 / 3$ and $2 / 3[\overline{2} 1 \overline{1}]_{\beta}$. Thus, the examination of the initial microstructures, and their respective stress-strain plots, suggests that PLC-like effect is suppressed in the equilibrium

$\alpha+\beta$ microstructure. In order to obtain a mechanistic understanding of the PLC effect in the $\beta 21 \mathrm{~S}$ the microstructure of deformed (at $300^{\circ} \mathrm{C}$ ) specimens aged at $350^{\circ} \mathrm{C} / 100 \mathrm{Hrs}$ (with high volume fraction of well-developed ellipsoidal $\omega$ precipitates) was examined in detail. 
Mechanically tested specimens were polished prior to testing in order to examine whether deformation bands were formed. SEM examination of the gauge section of the deformed specimens revealed presence of copious deformation bands, majority of which were approximately parallel to the plane of maximum shear stress (Fig. 2a). TEM foils were plucked from inside a $\beta$ grain, where the deformation bands are approximately at $45^{\circ}$ with respect to the tensile axis (depicted in the SEM image in Fig. 2a). The deformed microstructure, shown in the bright-field TEM (BFTEM) image in Fig.2b , consisted of two regions - a large deformation band along $\{112\}_{\beta}$ with crosshatched marks (dotted circle in Fig. 2b) and ones without those marks outside the deformation band (bottomright Fig. 2a).

A closer examination of the crosshatched marks (via BFTEM with $\mathrm{g}=[\overline{2} 00]_{\beta}$ near $[011]_{\beta}$ zone axis) revealed that such marks are micro-slip bands lying close to $\{011\}_{\beta}$ planes. Multiple variants of $\{011\}_{\beta}$ micro-slip bands, with very high dislocation density were seen within each deformation band suggesting that the extensive plastic flow was localised within the former. (Fig.2c). It may be pointed out that such micro-slip bands were also observed in the microstructure of the $\beta$-solutionized sample deformed at room temperature to $\sim 5 \%$ plastic strain. The BFTEM $\left(\mathrm{g}=[\overline{11} 0]_{\beta}\right.$ near $[001]_{\beta}$ zone axis) in Fig.2d, consisted of micro-bands lying close to $\{011\}_{\beta}$, and dislocation arrangements (arrow) depicting the early stages of the formation of micro-slip bands. Trace analysis and g.b examination indicated that the dislocations inside the micro-slip bands were 1/2 $\langle 111\rangle_{\beta}$. This specific $\beta$-solutionized condition (Fig.2d) allowed us to examine the $\beta$ matrix deformed microstructure (Fig.1d), by avoiding extraneous influences because of well-developed precipitates. Notwithstanding, both Figs $2 \mathrm{c}$ and $2 \mathrm{~d}$ indicated that the parent $\beta$ matrix locally deforms through the formation of smaller micro-slip bands inside a larger deformation band. Implication of these bands on the PLC-like effect will be discussed in a latter section.

The deformed $\beta$-soln $+350^{\circ} \mathrm{C} / 100 \mathrm{Hrs}$ specimen which underwent extensive plastic flow (due to straining to failure) and several discontinuous yielding events were further investigated with the aim of identifying features which distinguish the microstructures 
outside and inside the deformation band (DB) regions. Fig. 3a and $3 \mathrm{~b}$ compares the $[011]_{\beta}$ SADP recorded from the two regions using the same set of parameters, i.e. selected area aperture width, camera-length, condenser intensity and exposure time. This comparison shows that the intensity maxima at $1 / 3$ and $2 / 3[\overline{2} 1 \overline{1}]_{\beta}$ related to the $\omega$ phase, are significantly lower within the $\mathrm{DB}$ than outside $\mathrm{DB}$, whereas the intensity maxima corresponding to $1 / 2[\overline{2} 1 \overline{1}]_{\beta}$ reflection appears to be significantly higher within the DB compared to that in the region outside. The differences in the intensities was also quantified in Fig.3c, which suggested that the phase associated with $1 / 2[\overline{2} 1 \overline{1}]_{\beta}$ reflection, matching $\{10 \overline{1} 0\}_{\alpha}$ reflection, is present in higher volume fraction inside DB..

Precipitate distribution outside and inside the DB was further examined via DFTEM by selecting (dotted circles in Fig. $2 a$ and $2 b$ ) all four $\omega$ reflections and the intensity maxima at $1 / 2[\overline{2} 1 \overline{1}]_{\beta}$. The microstructure outside DB, Fig.3d, contained large volume fraction of coarse $(\sim 50 \mathrm{~nm}$ long) ellipsoidal $\omega$ precipitates and in some rare instances $\alpha$ platelets. This microstructure is comparable to that of the pre-deformed condition (Fig.1c), but precipitates appear to be coarser ( $\sim 50 \mathrm{~nm}$ compared to $\sim 30 \mathrm{~nm}$ in Fig.1c). In contrast, regions inside the DB (Fig.3e) contained fine scale $\alpha$ precipitates arranged in crosshatched features (indicated with dotted lines), $\alpha$ laths of varying size scales (ellipses), and a few $\omega$ precipitates. The significantly lower number density of $\omega$ precipitates inside DB compared to that outside DB (consistent with the $[011]_{\beta}$ SADP comparison presented earlier) points to the fact that the extensive plastic flow within the DBs has resulted in the "dissolution" of the $\omega$ precipitates.

In some cases, $\omega$ precipitates with "segmented" appearance were also observed inside DB (Fig.3e inset), with fine scale precipitates forming the background. This feature was further examined in detail by individually selecting $\omega$ and $1 / 2[\overline{2} 1 \overline{1}]_{\beta}$ reflections (using the smallest objective aperture) from the [011 $]_{\beta}$ SADP (circles in top-right insets in Fig.3f and $3 \mathrm{~g}$ ), and recording the corresponding DFTEM images i.e. Fig.3f and 3g. Selection of $1 / 3[\overline{2} 1 \overline{1}]_{\beta}$ illuminates only an elongated, irregular-shaped $\omega$ precipitate, while selecting $1 / 2[\overline{2} 1 \overline{1}]_{\beta}$ highlights the fine-scale precipitates in crosshatched arrangement in Fig.3f. The observation of such $\omega$ morphology (Fig.3f) is rather intriguing because it neither 
conforms to the typical ellipsoidal morphology (Fig.1b and 3d), nor does isothermal coarsening changes the $\omega$ morphology so drastically [13]. Therefore it seems reasonable to assume that interaction with dislocations during plastic deformation may have contributed to the altered $\omega$ morphology.

Focusing on the fine-scale precipitates; typically the reflection at $1 / 2[\overline{2} 1 \overline{1}]_{\beta}$ of a $[011]_{\beta}$ SADP can be indexed as the $\{10 \overline{1} 0\}$ type reflection of $h c p-\alpha$ phase. The presence of $\alpha$ inside the DB was further confirmed by recording the [111 $]_{\beta}$ SADP (bottom-left inset in Fig.3g) from the crosshatched region, exhibiting $1 / 2[\overline{1} 1 \overline{2}]_{\beta}$ reflections as expected from the Burgers OR. These observations indicate that the micro-slip bands (present as crosshatched regions) within the DB are decorated with predominantly a precipitates, and most of the fine $\omega$ precipitates have disappeared from these regions.

In order to examine whether these fine-scale $\alpha$ precipitates are associated with any solute enrichment / depletion atom probe tomography was performed on specimens, which were extracted from the same DB as indicated in Fig.2a. The atom probe reconstruction shown in Fig.4a, representing a small volume within the DB, shows $\omega$ precipitates delineated via a 3.5at\% Mo isoconcentration surface [14]. A comparable region in the deformed microstructure is indicated inside a DFTEM in Fig.4b. The region between the $\omega$ precipitate in Fig.4a was further subjected to cluster analysis [15], which revealed Alrich clusters of about $\sim 5-7 \mathrm{~nm}$ in diameter that matches the size scale of the fine $\alpha$ precipitates (Fig.3g. and 4b). The average composition of these Al-rich clusters, determined from the APT analysis software was 58.7Al-38.0Ti-2.4Mo-0.5Nb-0.2Si(at\%). While this composition is clearly not indicative of the composition of $\alpha$ precipitates, it presents experimental evidence of $\mathrm{Al}$ clustering within the DB.

The microstructural evidence collected precisely from the deformation bands allowed us to construct a simple micromechanical description of the deformation process occurring during the observed serrated plastic flow. Schematics presented in Fig.4d sequentially depict different stages of successive stress drop and rise in the stress-strain plots. 
Examination of the deformed microstructures showed that the macroscopic deformation bands (DBs) consist of several finer-scale $\{110\}_{\beta}$ micro- slip bands (Fig.2), within which fine $\omega$ particles are dissolved and coarse ones are segmented (Fig.3) at the onset of a stress drop. It is envisaged that a sudden stress drop occurs when, leading dislocations within a slip band create soft $\omega$-free channels, making way for the movement of an avalanche of dislocations leading to localized plastic flow,[1-4].. In an appropriate temperature-strain rate regime, a dynamic precipitation process can pin some of the mobile dislocations and restore the hardness of the soft channels. With a drop in the mobile dislocation density there is a necessity for the stress to increase to maintain the imposed strain rate. The interplay of these two competing processes results in the appearance of serrated yielding (PLC like effect). In case of $\beta$-Ti alloys the rapid pinning of dislocations is possible by dynamic precipitation of one of the competing phases, $\alpha$ or $\omega$, along the dislocation lines. Observation of $\alpha$ precipitates in the micro slip bands of deformed $\beta 21 \mathrm{~s}$, exemplified in Fig.3, demonstrates that the pinning of dislocations in the soft channels was not due to $\omega$ re-precipitation, as was observed in Ti-15 wt \% Mo [4]. The presence of $\mathrm{Al}$ and a higher stability of the $\alpha$ phase in preference to $\omega$ appears to be responsible for the difference in the dislocation pinning mechanisms in $\beta 21 \mathrm{~s}$ and Ti$15 \mathrm{wt} \%$ Mo. Moreover, the $\alpha$ precipitation, due to higher transformation strains than $\omega$ [3], is expected to strongly interact with dislocation stress fields. Dislocation mediated $\alpha$ precipitation has been observed earlier [16], and theoretically examined by Dui et.al. via phase-field modeling [17].

Results of the Atom Probe experiments have clearly shown that Al-rich clusters of sizes matching with that of $\alpha$ precipitates are present in the micro-slip bands. This suggests that enrichment of $\mathrm{Al}$ in such small regions has promoted $\alpha$ precipitation. A comparison of $\mathrm{Al}$ and Mo diffusion constants in $\beta$ Ti phase at $300^{\circ} \mathrm{C}$ shows that $\mathrm{Al}$ had a much higher diffusivity (diffusivities of $\mathrm{Al}$ and $\mathrm{Mo}$ are $5.15 \times 10^{-7}$ and $3.17 \times 10^{-11} \mu \mathrm{m}^{2} / \mathrm{s}$ respectively [18]) and can, therefore, restore hardness of the soft channels relatively faster by $\alpha$ precipitation. For example, within the time frame of an individual serration (inset Fig.1a ), i.e. 500-1000s, Al and Mo diffuses by 16-22 nm and 0.1-0.2 nm respectively at $300^{\circ} \mathrm{C}$, and the diffusion length of $\mathrm{Al}$ is either greater than or comparable 
to the size of observed $\alpha$ precipitates (Fig.3e). Since Al is a potent $\alpha$ stabilizer $[3,10]$, it is expected that pipe diffusion of $\mathrm{Al}$ along such dislocation line will promote dynamic nucleation of $\alpha$ nucleation on dislocations; leading to the hardening of the prior soft channels and the observed load-rise (Fig.1a and Fig.4).

Summarizing, Portevin-Le Chatelier or PLC-like effect, i.e. serrated plastic flow in the tensile response at elevated temperatures, was observed in a commercially important $\beta$ stabilized $\beta 21 \mathrm{~S}$ Ti-alloy. The repeated load-drops during the discontinuous yielding process have been found to be associated with the sudden localized plastic flow within the deformation bands. TEM studies of the deformation bands have revealed extensive dislocation activity within the $\omega$-free micro-slip-bands. The dynamic precipitation of $\alpha$ phase arrests the plastic flow in the $\omega$-free channels. The interplay of these two competing processes results in the PLC-like effect. The present results demonstrate that precipitate-dislocation interactions can also manifest PLC-like effect.

\section{Acknowledgements}

The authors would like to thank the funding sources NSF grant \#1309277 and \#1435611. We thank Prof R.S. Mishra at UNT for the utilization of his laboratory facilities. Additionally, Dr. Victor Ageh is thanked for preparing the FIB lift-outs. 


\section{References}

1. A. Portevin and F. Le Chatelier, C.r. Acad. Sci. 176 (1923) 057

2. G. E. Dieter: Mechanical Metallurgy, McGraw-Hill , 1988, pp.26-35 and 201-203,

3. S. Banerjee and P. Mukhopadhyay: Phase Transformations- Examples from Titanium and Zirconium alloys, $1^{\text {st }}$ ed, Pergamon, Great Britain, 2007

4. S. Banerjee and U.M. Naik, Acta. Mater., 44 (1996) 3667-3677

5. F. Popille, L.P. Kubin, J.Douin and S. Naka, Scripta. Mater., 34 (1996) 977-984

6. R.J. Grylls, S. Banerjee, S. Perungulam, R. Wheeler, and H.L. Fraser, Intermetallics, 6 (1998)749-752

7. A.M. Garde, A.T. Santhanam and R.E. Reed-Hill, Acta. Metall., 20 (1972) 215-220

8. K. Prasad, A.Amrithapandian, B.K. Panigrahi, V. Kumar, K.B. Sankara-. Rao and M. Sundararaman, Mater. Sci. Eng. A, 638 (2015) 90-96

9. M.J. Lai, C.C. Tasan and D. Raabe, Acta. Mater., 100 (2015) 290-300

10. G. Lutjering and J.C. Williams: Titanium, Springer, 1910, $2^{\text {nd }}$ ed

11. S.A. Mantri, D. Choudhuri, A. Behra, J.D. Cotton, N. Kumar, and R. Banerjee, Metal. Mater. Trans. A, 64A (2015) 2803

12. N. Kumar, D. Choudhuri, R. Banerjee and R. Mishra: Int. J. Plasticity, 68 (2014) 77 97

13. A. Devaraj, S. Nag, R. Srinivasan, R.E.A. Williams, S. Banerjee, R. Banerjee, H.L. Fraser, Acta Mater. 60 (2012) 596;S. Nag, R. Banerjee, R. Srinivasan, J.Y. Hwang, M. Harper, H.L. Fraser: Acta Mater., 57 (2009) 2136-214

14. O.C. Hellman, J.A. Vandenbrouucke, J. Rusing, D. Isheim, D.N. Seidman. Microsc. Micro-anal. 6 (2000) 437

15. J.M. Hyde, E. A. Marquis, K.B. Wilford and T.J. Williams, Ultramicroscopy, 111 (2011)440

16. N. Gey, M. Humbert, M.J. Philippe and Y. Combres, Mater Sci. Eng. A, A230 (1997)68-74; H. Moustahfd, M. Humbert and M.J. Philippe, Acta Mater. 45 (1997) 3750

17. D. Qiu, R. Shi, D. Zhang, W. Liu and Y. Wang, Acta. Mater. 88 (2015) 218-231

18. H.Araki, T. Yamane, Y. Minmino, S. Saji, Y. Hana and S.B. Jung, Metall. Mater. Trans. A, 25A(1994) 874; S.L. Semiatin, S.L. Knisley, P.N. Fagin, F. Zhang and Dr.R. Barker, Metall. Mater. Trans. A, 34A(2003) 2377 


\section{Figure Captions}

Figure 1. (a) Engineering stress-plastic strain plots of three different heat-treated conditions. Dark-field TEM (DFTEM) images and the corresponding [011 $]_{\beta}$ SADP of the initial microstructures after: (b) $\beta$-solutionized (c) $\beta$-solutionizing $+350^{\circ} \mathrm{C} / 100 \mathrm{Hrs}$ (d) $\beta$ solutionizing $+350^{\circ} \mathrm{C} / 100 \mathrm{Hrs}+600^{\circ} \mathrm{C} / 1 \mathrm{Hr}$ (top-right right inset $\mathrm{SEM}$ ).

Figure 2. (a) SEM image of a deformed $\beta$-solutionized $+350^{\circ} \mathrm{C} / 100 \mathrm{Hrs}$ specimen and the location of the TEM lift-out. (b) Low magnification BFTEM from the lift-out. (c) A magnified view of a region in " $b$ " showing crosshatched bands and (d) micro-slip trace in deformed $\beta$-solutionized sample.

Figure 3. Comparison of diffraction patterns from (a) outside and (b) inside a deformation band (DB), and (c) comparison of intensity profiles. DFTEM from (d) outside and (e) inside the DB. DFTEM examination of a region inside the deformation band by individually selecting reflections corresponding to (f) $\omega$ and (g) $\alpha$ phases in the $[011]_{\beta}$ SADP. Insets show $[011]_{\beta}$ SADP in the top-right of both "f" and "g" and $[111]_{\beta}$ SADP in the bottom left of "g").

Figure 4. (a) 3D reconstruction of APT tip, (b) a DFTEM of a comparable region, (c) Alrich cluster present in the APT reconstruction, and (d) schematic of mechanism for loaddrop and rise. 

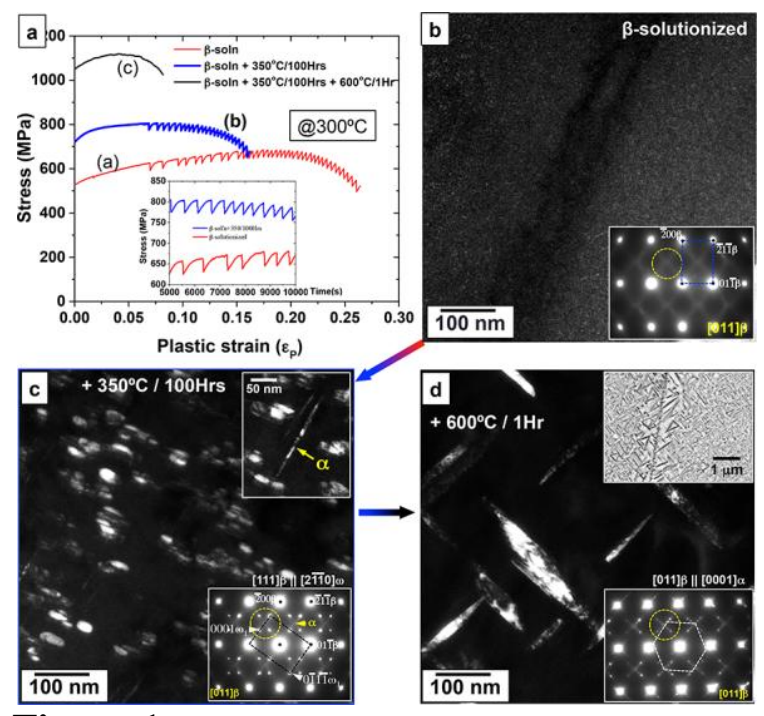

Figure 1 


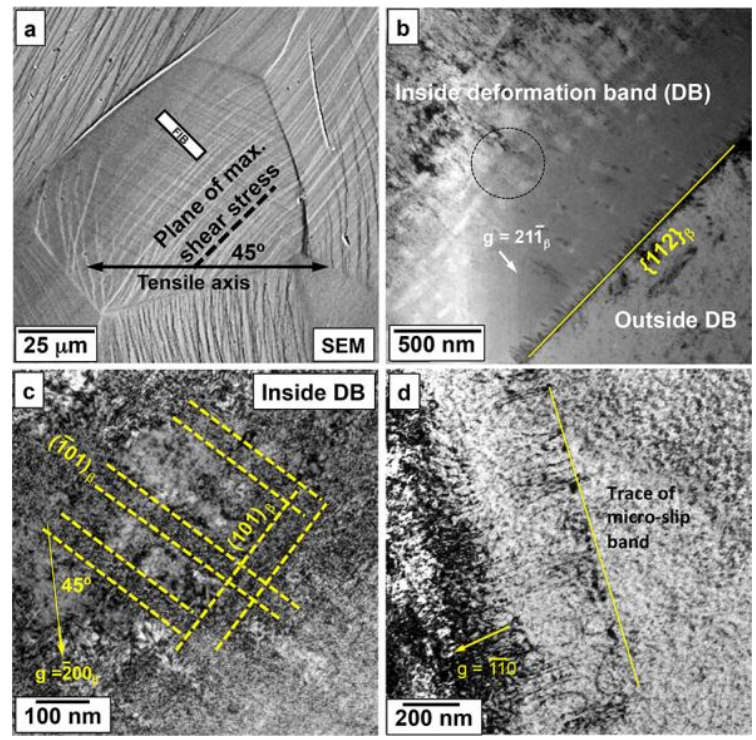

Figure 2 


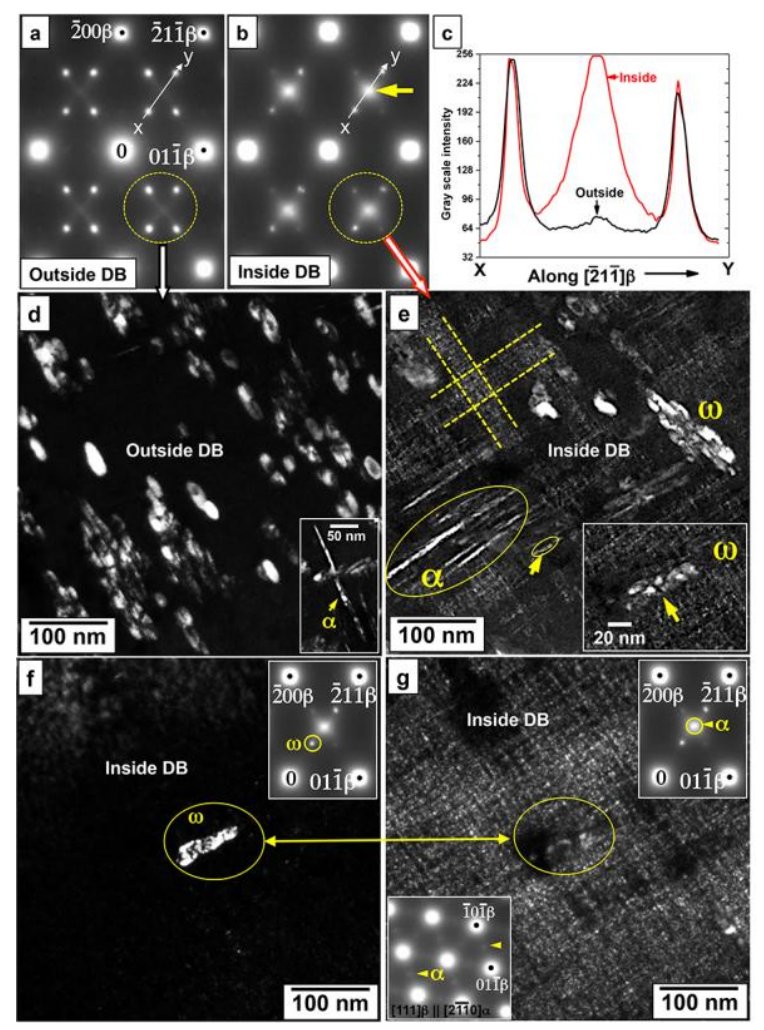

Figure 3 


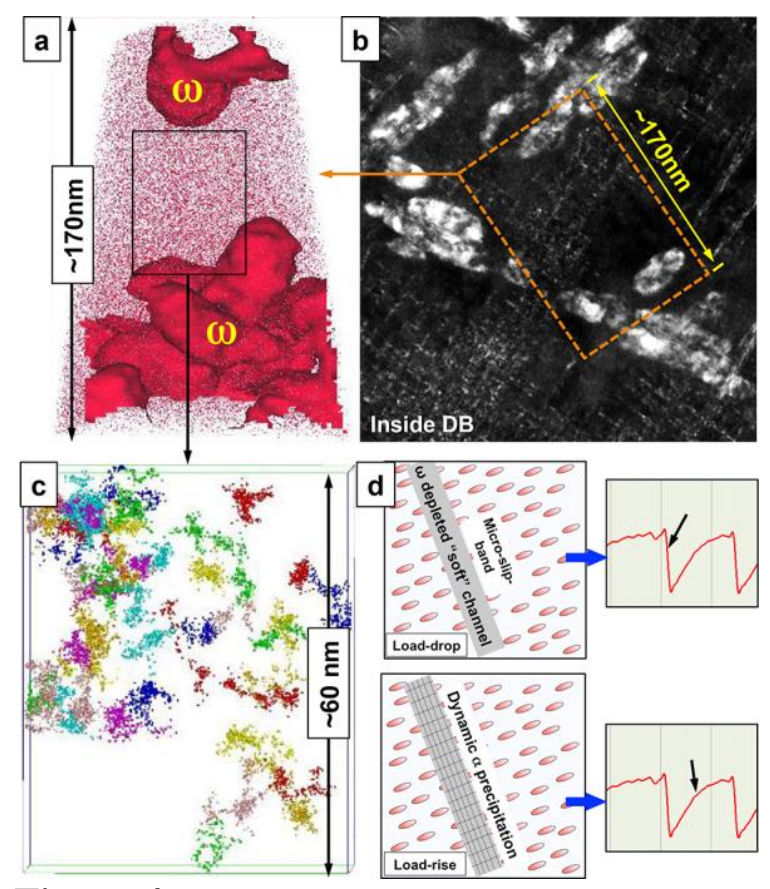

Figure 4 

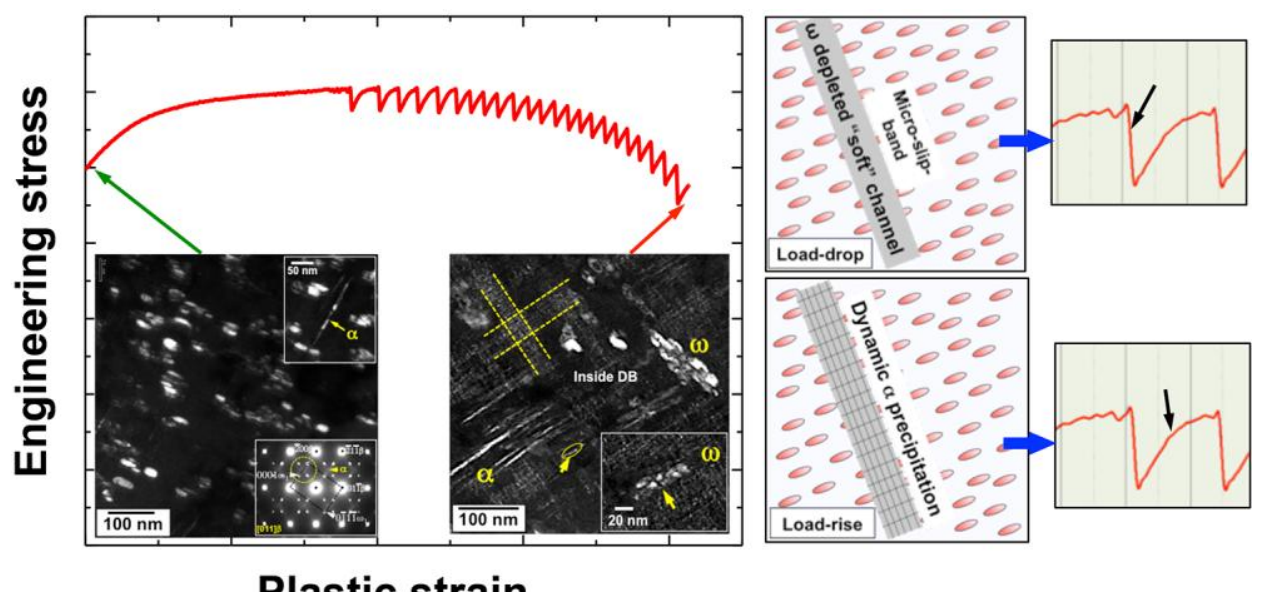

Graphical abstract 